Article

\title{
Fast Volumetric Ultrasound B-Mode and Doppler Imaging with a New High-Channels Density Platform for Advanced 4D Cardiac Imaging/Therapy
}

\author{
Lorena Petrusca ${ }^{1, *}$, François Varray ${ }^{2}$ (D), Rémi Souchon ${ }^{3}$, Adeline Bernard ${ }^{2}$, \\ Jean-Yves Chapelon ${ }^{3}$, Hervé Liebgott ${ }^{2}$, William Apoutou N'Djin ${ }^{3}$ (D) and Magalie Viallon ${ }^{1,2}$ \\ 1 University of Lyon, UJM-Saint-Etienne, INSA, CNRS UMR 5520, INSERM U1206, CREATIS, \\ F-42023 Saint-Etienne, France; magalie.viallon@creatis.insa-lyon.fr \\ 2 University of Lyon, UCBL, INSA, UJM-Saint Etienne, CNRS UMR 5520, INSERM U1206, CREATIS, \\ F-69100 Lyon, France; Francois.Varray@creatis.insa-lyon.fr (F.V.); Adeline.Bernard@creatis.insa-lyon.fr (A.B.); \\ Herve.Liebgott@creatis.insa-lyon.fr (H.L.) \\ 3 LabTAU, INSERM, Centre Léon Bérard, Université Lyon 1, Univ Lyon, F-69003 Lyon, France; \\ remi.souchon@inserm.fr (R.S.); Jean-Yves.Chapelon@inserm.fr (J.-Y.C.); apoutou.ndjin@inserm.fr (W.A.N.) \\ * Correspondence: Lorena.Petrusca@creatis.insa-lyon.fr; Tel.: +33-472431988
}

Received: 22 December 2017; Accepted: 25 January 2018; Published: 29 January 2018

\begin{abstract}
A novel ultrasound (US) high-channels platform is a pre-requisite to open new frontiers in diagnostic and/or therapy by experimental implementation of innovative advanced US techniques. To date, a few systems with more than 1000 transducers permit full and simultaneous control in both transmission and receiving of all single elements of arrays. A powerful US platform for implementing 4-D (real-time 3-D) advanced US strategies, offering full research access, is presented in this paper. It includes a 1024-elements array prototype designed for 4-D cardiac dual-mode US imaging/therapy and 4 synchronized Vantage systems. The physical addressing of each element was properly chosen for allowing various array downsampled combinations while minimizing the number of driving systems. Numerical simulations of US imaging were performed, and corresponding experimental data were acquired to compare full and downsampled array strategies, testing 4-D imaging sequences and reconstruction processes. The results indicate the degree of degradation of image quality when using full array or downsampled combinations, and the contrast ratio and the contrast to noise ratio vary from $7.71 \mathrm{~dB}$ to $2.02 \mathrm{~dB}$ and from $2.99 \mathrm{~dB}$ to $-7.31 \mathrm{~dB}$, respectively. Moreover, the feasibility of the 4-D US platform implementation was tested on a blood vessel mimicking phantom for preliminary Doppler applications. The acquired data with fast volumetric imaging with up to $2000 \mathrm{fps}$ allowed assessing the validity of common 3-D power Doppler, opening in this way a large field of applications.
\end{abstract}

Keywords: ultrasound; 4-D; cardiac; fast volumetric imaging; platform; advanced imaging; power doppler

\section{Introduction}

In the last 15 years, open research US machines [1] have been developed for therapy and imaging in order to offer different solutions to the ultrasound (US) community. Although the US imaging modality is considered a versatile, well-established and widely used diagnostic tool in medicine, novel approaches based on modern signal and image processing methods are required in order to improve the image quality and diagnostic accuracy. But the lack of flexibility offered by standard commercial systems, especially the limited access of RF raw data information and limited number of elements, does not always fit the requirements for data access and extensive control over imaging and systems parameters [2] and prevents a complete evaluation of new investigation methods [3]. Moreover, 3-D imaging systems provide a detailed view of tissue structures that make diagnosis 
easier for physicians. Therefore, volumetric US scanners with open access, that enable real time 3-D visualization of dynamic structures, including the heart, are required by the community.

A novel US open platform with high-channels density with optimal design constraints such as flexibility, precision, hardware efficiency, optimal acquisition architecture and wide access to RF data is a pre-requisite to open new frontiers in diagnostic and/or therapy by experimental implementation of innovative advanced US techniques: dual-mode US imaging/therapies in the heart, new approaches to study the myocardial tissue (structure/characterization), fast sparse array strategies [4,5], multi-line transmit (MLT) [6], adaptive beamforming, and powerful motion correction strategies, etc. [7-10]. This flexibility is not available in commercial equipment designed for clinical use.

Few experimental high research systems in the world [11,12] today permit full control both in transmission and receiving of all single elements simultaneously of arrays with more than 1000 transducers, requiring as many channels as the number of active elements. One of them, the SARUS scanner [12], is designed to allow full exploitation of 2-D ultrasound transducers, but heavy hardware is required, and therefore its portability is a weak point. In vivo 3-D results, including Doppler applications and cardiac fiber orientation, have already been published by using the parallelized Aixplorer systems $[13,14]$. For this setup, in order to synthetize a total of 1024 receiving channels, each emission must be repeated twice, with the receiving channels being multiplexed to 1 of 2 transducer elements. Therefore, this platform is not completely open and it has limited flexibility.

Other approaches have been investigated to allow addressing a large amount of active elements with a reduced number of channels: multiplexing [15] by using capacitive micromachined ultrasonic transducer (CMUT) technology [16], micro-beamforming [17] and row-column addressing [18]. But in this case, the flexibility of the sequence acquisition is reduced while the elements coupled in sub-arrays are not continuously connected to the US scanner, limiting the modularity of the system. Furthermore, 2-D optimized sparse arrays strategies were also proposed and evaluated, a continuous one-element-to-one-channel connectivity being provided [5].

The quantitative applications like tissue Doppler imaging or strain imaging when using conventional echocardiography used in clinical routine are limited due to the low frame-rate acquisitions. High frame-rate ultrasound imaging (also called ultrafast) represents a modality developed in the last fifteen years due to its great interest and numerous possible applications [9]. Around 150 frames per second are available when using conventional imaging techniques, while a frame-rate of more than 1000 frames per second is available with plane wave imaging [19]. The interest of achieving a high frame-rate in 3-D echography is highly important for clinical diagnosis, especially for moving organs like the heart. By improving the temporal resolution of the acquired images, the different phases of the cardiac cycle can be visualized and quantitative structural and functional information analyzed. Different methods based on focused or unfocused transmit beams were developed for ultrafast echocardiography, including plane waves (PW) [20], diverging waves (DW) [21] or MLT [6,22] approaches. For increased contrast and spatial resolution, the combination of several images is used for these techniques. Therefore, in this case, motion compensation algorithms $[23,24]$ are required to increase the image quality affected by large tissue displacements. Moreover, complex blood flow quantification [25] or artery elasticity assessment [26] could also be analyzed with ultrafast methods. When using 3-D field of view at a high frame-rate, full quantitative Doppler flow analysis can be performed on a large region of interest, leading to much more information and improved functionality for the clinician [27]. In addition, the accuracy of 3-D power Doppler can also be analyzed.

This paper presents a new powerful fully programmable US platform developed with specific performance for implementing 4-D (real-time 3-D) advanced US strategies with custom data processing. It includes 4 US systems with 256 channels each synchronized together and a US probe with a matrix array of $32 \times 32$ elements. The main features of this imaging system include its capability of simultaneous arbitrary waveform generation, full access to the RF data and the possibility of performing 4-D dual-mode US imaging and/or therapy. In addition, the platform is fully modular up to 1024 elements in full or downsampled regular or sparse array, portable and appropriate for diagnostic and/or therapy purposes. The technical implementation and validation of this platform 
is described here. Moreover, the feasibility of the fast volumetric US B-mode and Power Doppler imaging approach is also described, opening a large field of vascular and cardiac applications.

\section{Materials and Methods}

\subsection{US Experimental Platform}

The new ultrasound platform includes a 1024-element US probe with a specific wiring of the elements and a high-channels density US scanner driven by 4 individual Vantage 256 systems (Verasonics, Kirkland, WA, USA), synchronized together. The development of this US platform was possible thanks to a close collaboration between two laboratories, by sharing together the required materials (the US probe and four US systems) and their competences. The materials were used together for all the experiments presented in this work.

US probe. The customized US probe (Vermon, Tours, France) (Figure 1d) for cardiac applications is a $32 \times 35$ matrix array with a $0.3 \mathrm{~mm}$ pitch in both $x$ and $y$ directions and a square footprint with sides of about $10 \mathrm{~mm}$. In the $y$ direction, the 9th, 17th and 25th lines are not connected, resulting in a total number of 1024 active elements.

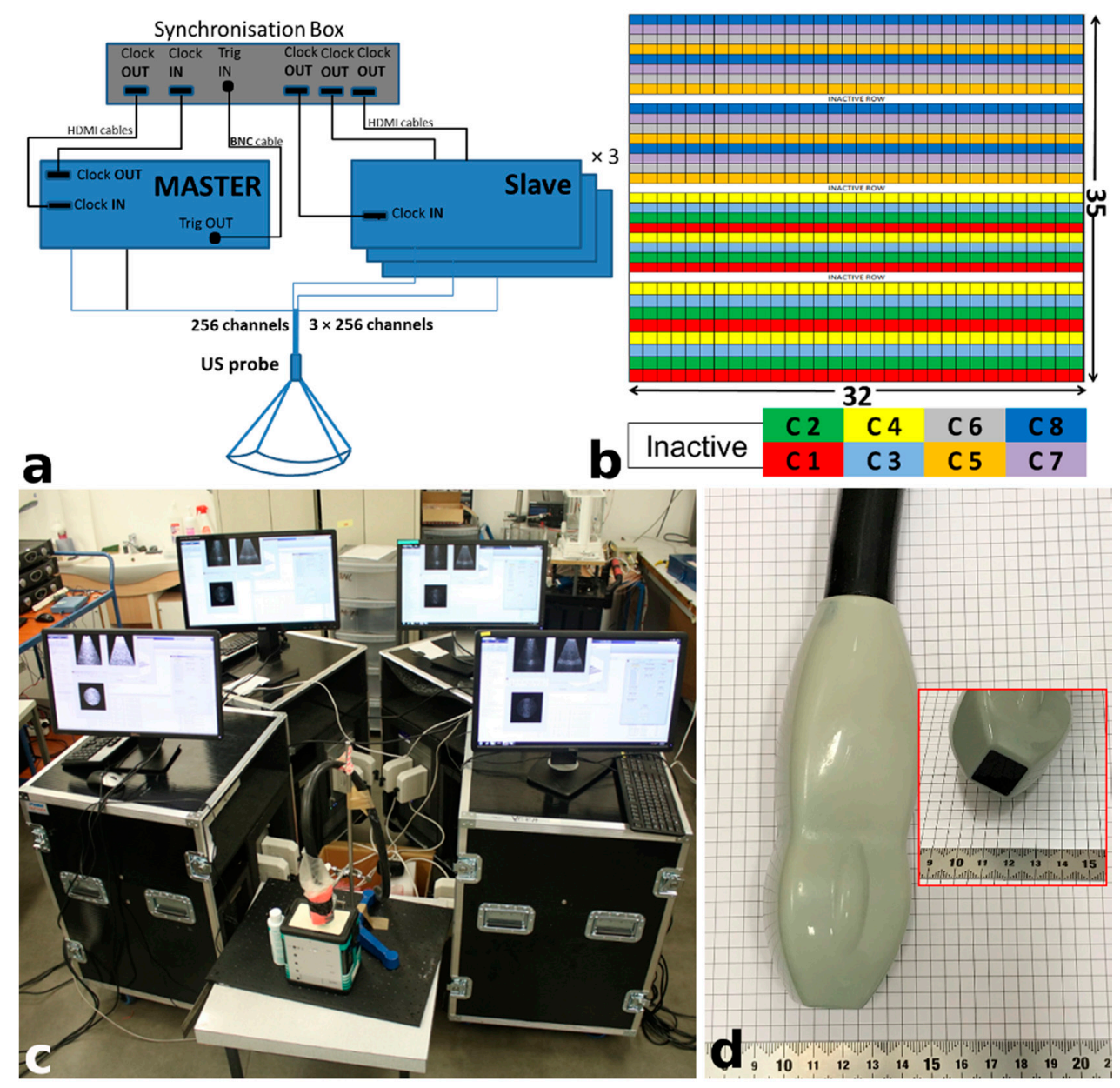

Figure 1. (a) Block diagram of the entre setup including the Master, the 3 Slaves, the synchronization box and the US probe; (b) Diagram of the 1024-element distribution across 8 connectors. Each color corresponds to a connector (C); (c) Picture of the 4 synchronized Vantage systems and the 3-D US probe connected; (d) the customized 3-D US cardiac probe.

The 1024 elements of the probe were carefully spread out across the 4 systems, with 2 connectors per system, each element being physically connected to the same single channel both in transmission and reception (one-element-to-one-channel design). The physical addressing of each US element was 
specifically chosen for allowing various array sparsity combinations while minimizing the number of required driving systems. A detailed diagram illustrating the element distribution is shown in Figure $1 \mathrm{~b}$ ). Indeed, each connector of 128 channels controls 4 entire rows (of 32 elements each) distributed across every 4 rows in the matrix. In this way, for a full active array, 4 systems are required, but regular or irregular sparsity of the US array can be activated using a lower number of US systems. All active channels can be used simultaneously in transmission and reception. Moreover, the connectors incorporate impedance matching circuits for power transmission, if required. The central frequency was chosen as $3 \mathrm{MHz}$ with a $70.9 \%$ bandwidth of $-6 \mathrm{~dB}$.

Therefore, the physical addressing of the US prototype was determined as a compromise between the simplicity of internal connection, flexibility for testing various downsampled configurations and the reduction of the number of systems needed. The interest of several simple sparsity configurations to generate exploiTable 4-D US images was investigated in simulation and then validated experimentally.

Ultrasound systems. The Vantage 256 scanners US systems (Verasonics, Kirkland, WA, USA) drive 256 elements each ( 2 connectors of 128 elements per board in each system) and are a research system entirely programmable (data acquisition and processing platform), designed to allow the development of new advanced US imaging strategies. They have per-channel arbitrary waveform transmit/receive generation capability with full access to RF data from every channel. The systems can be controlled by using Matlab through open user interface, allowing access to RF data of all channels and in particular, to display reconstructed data for ultrasound guidance in real time and offering extreme flexibility and precision. Standard or customized US transducers arrays can be connected to the systems, after declaration of the specifications of the US probe.

Synchronization box. A special Multi-System Synchronization Module capable of distributing the Master system clock to up to 8 Vantage systems via common HDMI cables designed and commercialized by Verasonics was used. One of the 4 systems is the "Master", providing the $250 \mathrm{MHz}$ clock, trigger and other signals for accurate temporal synchronization. Therefore, the module triggers in transmission and reception all the connected systems (considered as "Slaves") to co-start the acquisitions, with a less than 10 ns delay. Parallel acquisition can then be performed, each system collecting the data from the 256 elements of the connected sub-probe. This method allows control of up to 1024 elements at once. A block-diagram of the entire setup is illustrated in Figure 1a, while a picture of the experimental setup is presented in Figure 1c.

\subsection{Numerical Simulations}

Numerical simulations (in Field II simulation Program [28,29]) of US imaging were performed first in order to test various configurations and appropriately choose the best ones for the final US probe design. The possible configurations of the US matrix probe, including full and regular downsampled arrays, were tested and the image quality analyzed. The media whose images have been simulated included (i) equidistant point markers, (ii) a cyst or (iii) a numerical phantom with 500,000 scatters having uniform spatial distribution and amplitudes determined from an MR image of the human heart.

\subsection{Initial Experimental Acquisitions for Validation of the Platform}

The 4 Vantage systems were synchronized together for data acquisitions in 6 different array configurations allowed by the US probe. The validation of the setup was performed on a standard phantom (Figure 2) by using PW and DW. The used US acquisition parameters are described in Table 1. 
Table 1. US acquisition parameters.

\begin{tabular}{cc}
\hline Parameters & Value \\
\hline $\begin{array}{c}\text { Probe parameters } \\
\text { Matrix probe number of elements }\end{array}$ & $32 \times 32$ \\
\hline Pitch & $0.3 \mathrm{~mm}$ \\
\hline Center frequency & $3 \mathrm{MHz}$ \\
\hline $\begin{array}{c}\text { Imaging parameters } \\
\text { Transmit center frequency }\end{array}$ & $3 \mathrm{MHz}$ \\
\hline Sampling frequency & $12 \mathrm{MHz}$ \\
\hline Max imaging depth & $60 \mathrm{~mm}$ \\
\hline $\begin{array}{c}\text { Transmit full aperture } \\
\text { Pulse Repetition Frequency (PRF) }\end{array}$ & $4 \times 256 \mathrm{elements}$ \\
$200-2000 \mathrm{~Hz}$ \\
\hline Frame-rate & $200-2000 \mathrm{fps}$ \\
\hline
\end{tabular}

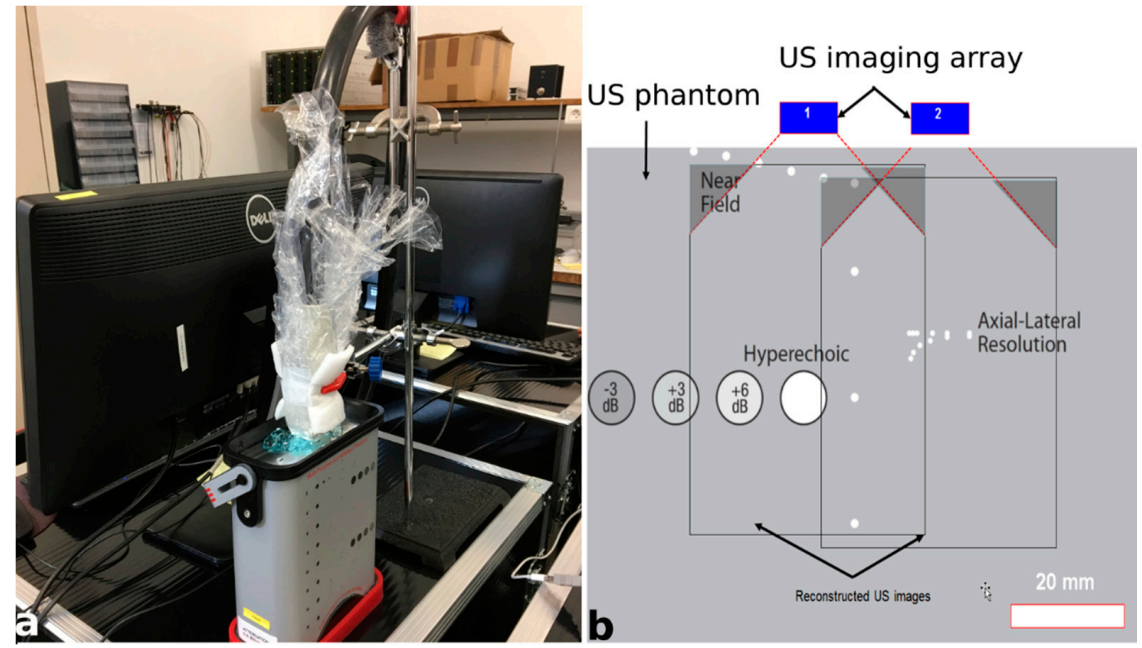

Figure 2. (a) Validation setup on a standard cyst phantom; (b) The diagram of the standard phantom used for experiments. 1 and 2 indicates 2 possible positions of the probe.

Table 2 presents all tested matrix configurations of the US probe for data acquired both with transmissions of plane waves (PW) and diverging waves (DW). The numbers of the connectors (C) are from 1 to 8 , while the Verasonics (V) systems are numbered from 1 to 4 . For symmetry reasons regarding the center of the probe along the $y$ direction, for the presented examples, the connectors are plugged as follows: $\mathrm{C} 4$ and $\mathrm{C} 5$ on V1, C3 and $\mathrm{C} 6$ on V2, C2 and C7 on V3, while C1 and C8 on V4.

Table 2. US probe matrix configurations and Verasonics combinations.

\begin{tabular}{ccccc}
\hline Array Configuration & $\begin{array}{c}\text { Matrix } \\
\text { \# Columns } \times \text { \#Rows }\end{array}$ & $\begin{array}{c}\text { No. of Active } \\
\text { Elements }\end{array}$ & $\begin{array}{c}\text { Connectors (C) } \\
\text { Used }\end{array}$ & $\begin{array}{c}\text { No. of Verasonics (V) } \\
\text { Systems }\end{array}$ \\
\hline Full array & $32 \times 32$ & 1024 & $\mathrm{C} 1: \mathrm{C} 8$ & $\mathrm{~V} 1: \mathrm{V} 4$ \\
1 line ON/1 line OFF & $32 \times 16$ & 512 & $\mathrm{C} 2, \mathrm{C} 4, \mathrm{C} 5, \mathrm{C} 7$ & $\mathrm{~V} 1, \mathrm{~V} 3$ \\
2 lines ON/2 lines OFF & $32 \times 16$ & 512 & $\mathrm{C} 2, \mathrm{C} 4, \mathrm{C} 5, \mathrm{C} 7$ & $\mathrm{~V} 1, \mathrm{~V} 2$ \\
4 lines ON/4 lines OFF & $32 \times 16$ & 512 & $\mathrm{C} 1: \mathrm{C} 8$ & $\mathrm{~V} 1: \mathrm{V} 4$ \\
Middle ON & $32 \times 16$ & 512 & $\mathrm{C} 1: \mathrm{C} 8$ & $\mathrm{~V} 1: \mathrm{V} 4$ \\
1 line ON/3 lines OFF & $32 \times 8$ & 256 & $\mathrm{C} 2, \mathrm{C} 7$ & $\mathrm{~V} 2$ \\
\hline
\end{tabular}

For the validation of the US platform, serial experiments were performed with PW and DW with 9 transmission waves ( 9 in the $x$ and $y$ direction, resulting in a total of 81 transmissions) by using the matrix 
configurations described before. An excitation of $30 \mathrm{~V}$ was applied in all cases. The US probe was maintained in a fixed position in order to strictly image the same volume of the phantom for a correct comparison of the image quality obtained with the different configurations. Further image quality analysis and the presence of secondary lobes were carried out to compare the results obtained with all configurations. For a qualitative evaluation of the results, the axial and lateral resolution was measured in $(x, z)$ and $(y, z)$ central slices of the phantoms. The full width at half maximum (FWHM) for 3 different wires positioned at 10, 20 and $30 \mathrm{~mm}$ was computed and the obtained values analyzed. Moreover, the contrast ratio (CR) and the contrast to noise ratio (CNR) were also analyzed, transformed to decibel (dB) scale. The CNR is a measure of the signal level in the presence of noise, expressing the fact that detectability increases with increasing object contrast and decreasing acoustic noise. An inside region was defined in a $(y, z)$ plane as a circle of radius $6.6 \mathrm{~mm}$ edge inside the cyst ( $8 \mathrm{~mm}$ diameter cyst), while an outside region of the same dimensions was chosen above the cyst. The same inside and outside regions were preserved for all the measurements. The CR and CNR were computed according to:

$$
\begin{gathered}
\mathrm{CR}=20 \log _{10}\left(\frac{\mu_{\text {in }}}{\mu_{\text {out }}}\right) \\
\mathrm{CNR}=20 \log _{10}\left(\frac{\left|\mu_{\text {in }}-\mu_{\text {out }}\right|}{\sqrt{0.5 \times\left(\sigma_{\text {in }}^{2}+\sigma_{\text {out }}^{2}\right)}}\right)
\end{gathered}
$$

where $\mu_{\text {in }} / \mu_{\text {out }}$ and $\sigma_{\text {in }} / \sigma_{\text {out }}$ correspond to the respective mean and standard deviation of the beamformed signals (of the B-mode image module) values inside/outside the cyst.

\subsection{Experimental Data Acquisitions for Doppler Application}

The feasibility of the 4-D platform implementation was tested on a blood vessel mimicking phantom [30] for 3-D Doppler applications. The phantom is made in polyvinyl alcohol (PVA), silica and distilled water (10\%, $1 \%$ and $89 \%$ in weight, respectively), ensuring elasticity (from the PVA) and a good visualization (from the silica) in US images, with 5 cycles of freeze-thaw. The length of the phantom was around $8 \mathrm{~cm}$, while the phantom thickness and internal diameter were $2 \mathrm{~mm}$ and $8 \mathrm{~mm}$, respectively. A circulating liquid (water and corn flour) obtained by using a setup with an external pump mimicked the blood flow inside the vessel. The role of the corn flour dispersed in the water increased the blood backscattering in order to quantify the flow. The resulting phantom is close to biological tissues in terms of acoustic and mechanical characteristics [31] and the fluid is in accordance with the standard physical and acoustic properties of blood [32].

The phantom was immersed in static water for acoustic coupling, and the US cardiac probe described in Section 2.1 was placed a few centimeters above. Different set-ups between the vessel mimicking phantom and the US probe were investigated: US probe parallel to the tube phantom $(\theta=0)$ and tilted at an angle of $12^{\circ}$.

The acquisitions for 3-D Power Doppler evaluation were performed using the full active 1024 matrix array of the US probe, with a single PW transmitted. The volumes were acquired at a high frame-rate with $2000 \mathrm{~Hz}$ for each configuration.

\subsection{Data Processing}

The RF data were acquired individually for each of the 4 US systems from the active elements to which it was connected to. After the acquisition, the data were collected and merged together, carefully taking into account the inactive line distribution of the US probe in each system. One large matrix containing the information of all the active elements (up to 1024 elements) was obtained and the beamformed RF data were post-processed offline [33]. A dataset for an entire volume was further provided.

For Power Doppler visualisation of the images, 3D slicer software [34] was used, allowing 3-D view rendering, and the animations are shown in supplementary material. 


\section{Results}

\subsection{Preliminary Numerical Simulations}

Preliminary numerical simulations allowed estimating the degradation of the images induced by several levels of sparsity (or matrix covering) implemented in 1-D. Figure 3 illustrates 5 different configurations of the active elements, including the full active array and downsampled array with 512 or 256 elements, in the $(y, z)$ plane. The central slice of the simulated volume is illustrated here. As expected, the full array (a) leads to the best visual image quality, while for the configuration with only 256 active elements (only 1 US system required), the image quality is very poor (e) with large secondary lobes and poor contrast. This result is also confirmed by axial resolution measurements in the $(x, z)$ and $(y, z)$ planes, displayed in Table 3. This step allowed us to define the best configuration of the final US probe design. It can be noticed that, when the 512 elements are positioned with an important pitch between the active lines (configuration 4 lines ON/4 lines OFF), the overall slice quality is decreased in the $(y, z)$ plane compared to the two other 512 arrays.

Table 3. Axial resolution (the mean value) measured in $(x, z)$ and $(y, z)$ planes for the equidistant scatters when using different configurations of the matrix array.

\begin{tabular}{ccccccc}
\hline \multicolumn{2}{c}{ Axial Resolution (mm) } & Full Array & $\begin{array}{c}\text { 1 Line ON/1 } \\
\text { Line OFF }\end{array}$ & $\begin{array}{c}\text { 4 Lines ON/4 } \\
\text { Lines OFF }\end{array}$ & $\begin{array}{c}\text { 2 Lines ON/2 } \\
\text { Lines OFF }\end{array}$ & $\begin{array}{c}\text { 1 Line ON/3 } \\
\text { Lines OFF }\end{array}$ \\
\hline \multirow{2}{*}{$(y, z)$ plane } & $40 \mathrm{~dB}$ & 1.2 & 2 & 4.6 & 2.6 & 2.2 \\
& $-6 \mathrm{~dB}$ & 0.7 & 0.8 & 0.9 & 0.6 & 0.6 \\
\hline \multirow{2}{*}{$(x, z)$ plane } & $40 \mathrm{~dB}$ & 1.2 & 1.2 & 1.2 & 1.2 & 1.2 \\
& $-6 \mathrm{~dB}$ & 0.6 & 0.6 & 0.5 & 0.6 & 0.5 \\
\hline
\end{tabular}

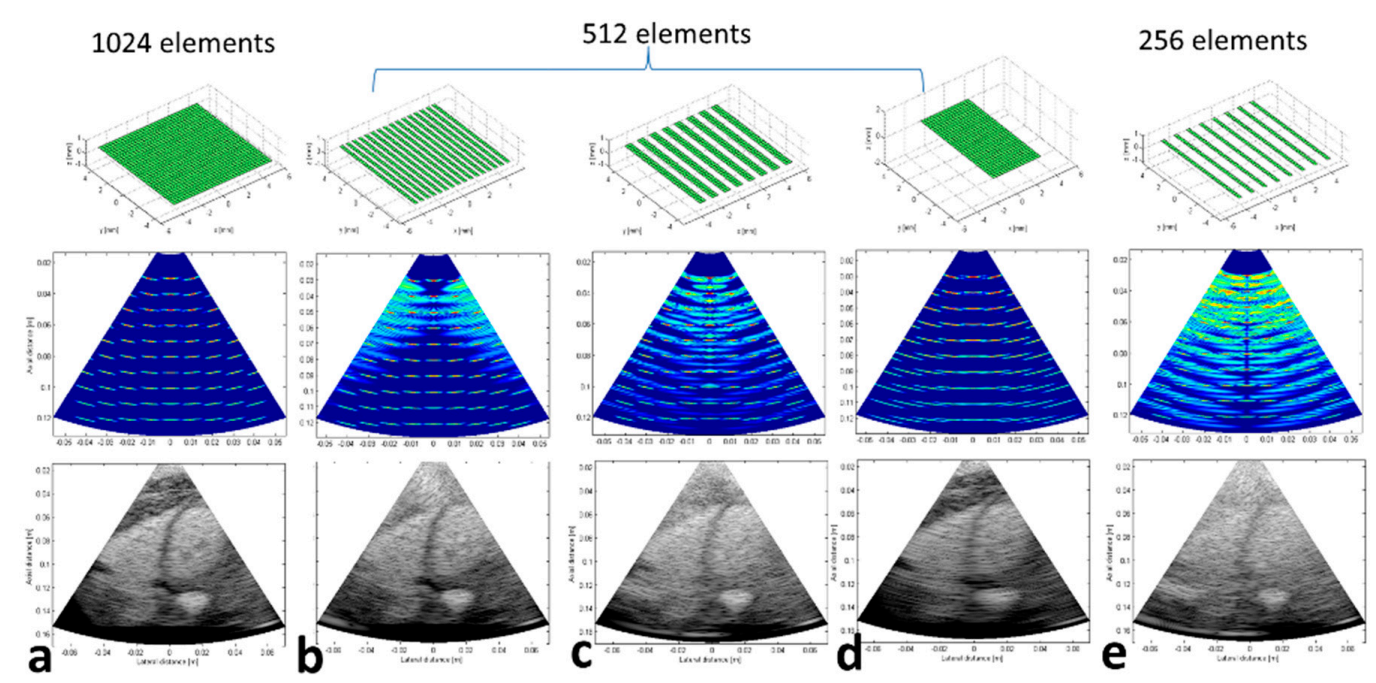

Figure 3. Numerical simulations results from Field II on equidistant scatters (2nd row) and based on an MR image of the heart (3rd row) corresponding to different configurations of the US array (1st row) in $(y, z)$ plane.

\subsection{Experimental Validation of the 4-D US Platform Developped}

An example of two different 3-D volumes obtained experimentally with 1024-channels US platform is illustrated in Figure 4). Data were obtained on a standard phantom (Gammex Sono410 SCG, schema illustrated Figure $2 b)$ with a cyst and a "grappe" and the orthogonal $(x, z)(a)$ and $(y, z)(b)$ planes are shown. The planes are taken in the center of the transducer's array, at $x=0 \mathrm{~mm}$ or $y=0 \mathrm{~mm}$, respectively. $9 \mathrm{PW}$ were used for the acquisition illustrated in the first row, while $9 \mathrm{DW}$ were used for the acquisition shown in the lower row. 

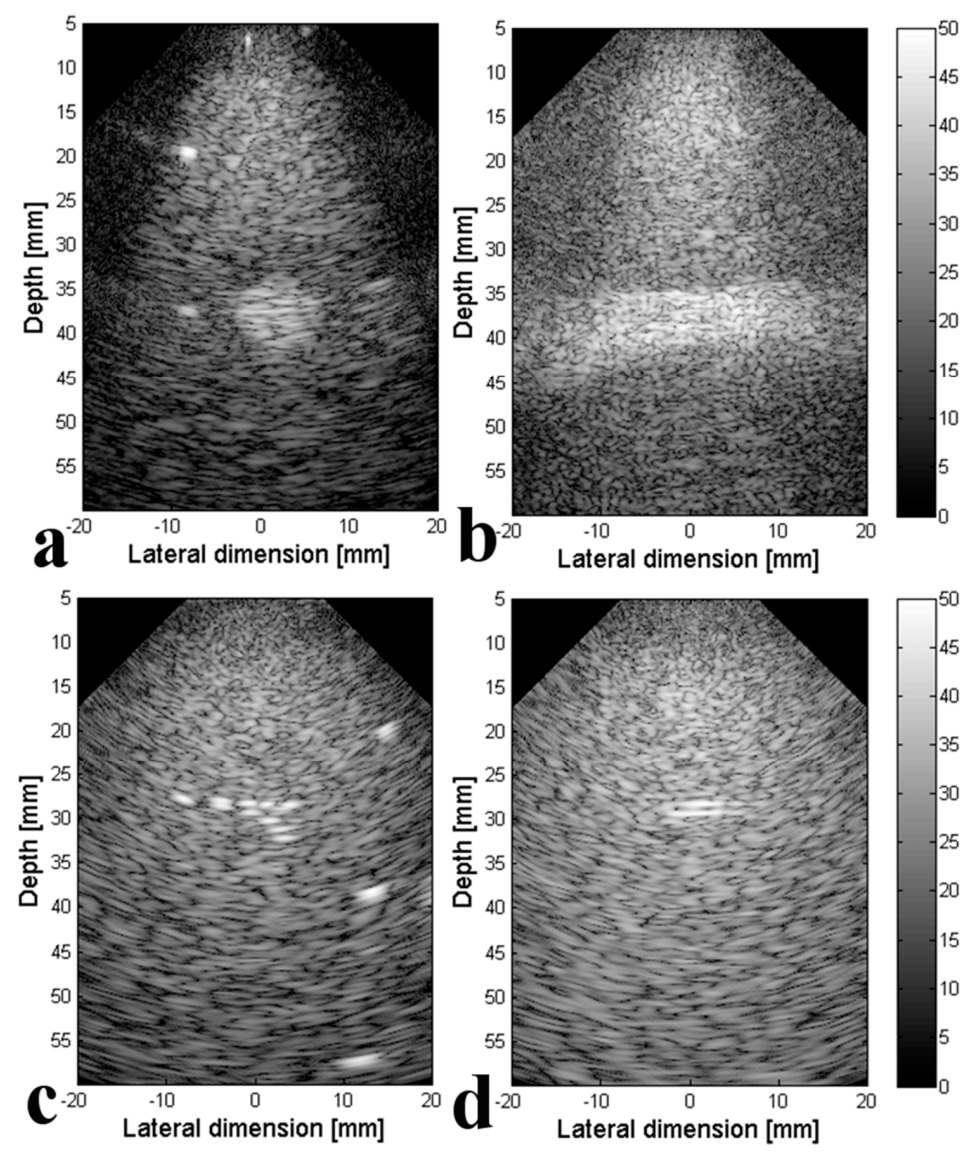

Figure 4. Experimental data obtained for the Gammex phantom with 1024 active elements in $(x, z)(\mathbf{a}, \mathbf{c})$ and $(y, z)(\mathbf{b}, \mathbf{d})$ planes on a cyst $(\mathbf{a}, \mathbf{b})$ by using 9 PW and "grappe" wires (c,d) by using 9 DW. A dynamic of $50 \mathrm{~dB}$ has been used in all images.

Different configurations of the US matrix array, by using 256, 512 or 1024 elements, were also investigated using the 4-D US platfom described above. Figure 5 illustrates an example of volumetric acquisition on a standard phantom with several wires positioned at different depths. Nine DW were transmitted in each of the $x$ and $y$ dirrections for this example. The $(x, z)$ and $(y, z)$ planes are shown here, the planes being taken at the center of the active array. As we expected, after visual inspection, the experimental results obtained here clearly indicate the full active array of the US probe as the best configuration. At a rapid inspection, the $(x, z)$ plane indicates similar results, but at a closer view, some differences are identified. The wire positioned in the near field (depth $=10 \mathrm{~mm}$ ) is very accurate with this configuration, while the configurations with 512 active elements still illustrate good accuracy. The 256-elements configuration (the last column) presents the worst case, as expected, taking into account the low number of active elements. The same observations are done if we inspect the wire situated in the far-field (depth $=50 \mathrm{~mm}$ ), where the best accuracy is visible in the full active array configuration.

At the same time, the $(y, z)$ plane illustrates the degradation of the image quality with lower numbers of active elements. The step in the $y$ direction is modified according to the configuration used, this leading to apparition of important artefacts for some cases, in the near and far-field. Not all the lengths of the wires are visible in this plane, due to small misalignment between the US probe and the wires. 


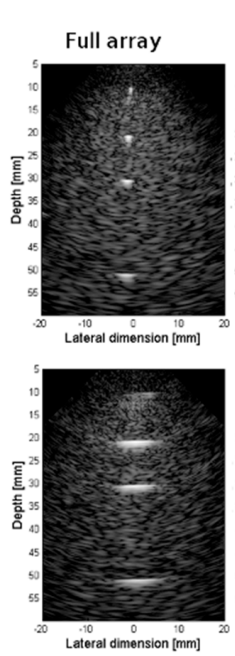

1024 elements
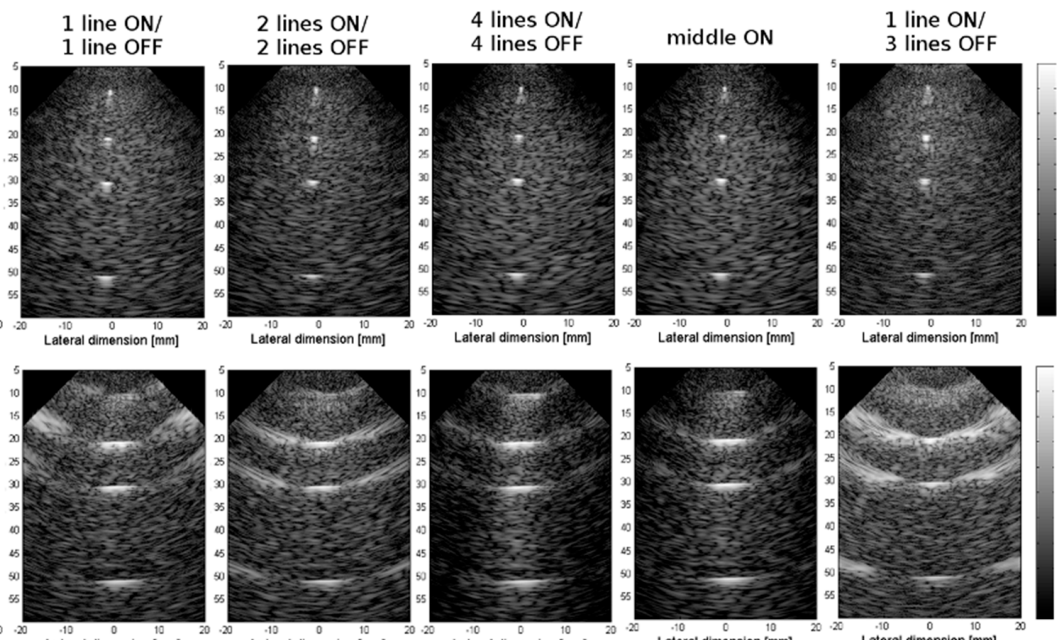

Lateral dimension $[\mathrm{mm}]$

Lateral dimension [mm

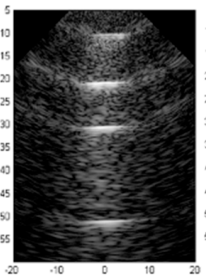

512 elements

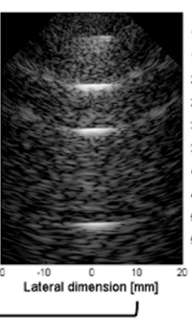

Lateral dimension $[\mathrm{mm}]$

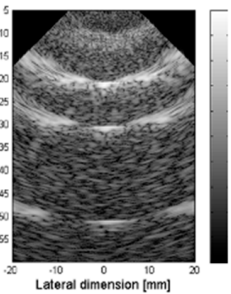

256 elements

Figure 5. Experimental data acquired with $9 \mathrm{DW}$ on a standard phantom. The $(x, z)$ plane (first row) and the $(y, z)$ plane (second row) are shown in the center of the US device. Different configurations of the array were used: full array, 1 line ON-1 line OFF, 2 lines ON-2 lines OFF, 4 lines ON-4 lines OFF, middle $\mathrm{ON}$ and 1 line ON-3 lines OFF. A dynamic of $50 \mathrm{~dB}$ has been used in all the images.

Further quantitative results of FWHM are measured and displayed in Table 4 , for the $(x, z)$ plane, in axial and lateral direction. These results confirm the previous observations, indicating the degree of degradation of the image quality when downsampled configurations are used.

Table 4. FWHM calculated in the $(x, z)$ plane over three scatters located at depths $10,20,30 \mathrm{~mm}$ and the CR and CNR evaluated on the $(y, z)$ central slices of the acquired volumes.

\begin{tabular}{|c|c|c|c|c|c|c|c|}
\hline \multicolumn{2}{|c|}{ Resolution (mm) } & Full Array & $\begin{array}{c}1 \text { Line ON/1 Line } \\
\text { OFF }\end{array}$ & $\begin{array}{l}2 \text { Lines ON/ } \\
2 \text { Lines OFF }\end{array}$ & $\begin{array}{l}4 \text { Lines ON/ } \\
4 \text { Lines OFF }\end{array}$ & Middle ON & $\begin{array}{l}1 \text { Line ON/ } \\
3 \text { Lines OFF }\end{array}$ \\
\hline \multirow{5}{*}{$(x, z)$ plane } & axial $20 \mathrm{~mm}$ & 1.7 & 2.5 & 3.1 & 2.2 & 2.3 & 3.3 \\
\hline & Average & 1.8 & 2.5 & 2.5 & 2.2 & 2.5 & 3.3 \\
\hline & lateral $10 \mathrm{~mm}$ & 0.9 & 1.6 & 1.7 & 0.9 & 1 & 2.3 \\
\hline & lateral $20 \mathrm{~mm}$ & 3 & 2.9 & 2.8 & 1.9 & 2.9 & 3.1 \\
\hline & lateral $30 \mathrm{~mm}$ & 2 & 2.9 & 3 & 3.1 & 3 & 3.4 \\
\hline \multirow{2}{*}{\multicolumn{2}{|c|}{$\begin{array}{c}\text { CR (dB) } \\
(y, z) \text { plane }\end{array}$}} & Full Array & 1 line $O N / 1$ line OFF & 2 lines $\mathrm{ON} / 2$ lines OFF & 4 lines ON/4 lines OFF & Middle ON & 1 line $\mathrm{ON} / 3$ lines $\mathrm{OFF}$ \\
\hline & & 7.71 & 2.84 & 2.30 & 4.20 & 3.50 & 2.02 \\
\hline \multicolumn{2}{|c|}{ CNR (dB) } & Full array & 1 line ON/1 line OFF & 2 lines $\mathrm{ON} / 2$ lines OFF & 4 lines ON/4 lines OFF & Middle ON & 1 line $\mathrm{ON} / 3$ lines OFF \\
\hline \multicolumn{2}{|c|}{$(y, z)$ plane } & 2.99 & -5.36 & -6.54 & -2.61 & -4.25 & -7.31 \\
\hline
\end{tabular}

Another example of the 9 DW acquisition with the same configurations of the US transducer is shown in Figure 6. A "grappe" wire was imaged in this standard phantom. The same general observations can be done as in the previous example. Additionally, in the $(x, z)$ plane, the separation of 2 different wires positioned very close to each-other (at depth $=28 \mathrm{~mm}$ ) is very hard to identify, except for the case of the full active array (first column). Moreover, the contour of the wires positioned at the border of the image, not directly in front of the US probe, are very hard to identify for configurations with a lower number of elements. Important secondary lobes and image degradation is visible in the $(y, z)$ planes illustrated here. The two parallel lines are clearly distinguished when using 1024 elements, but for 256 elements and even for some cases with 512 elements, it is hard to define them. 


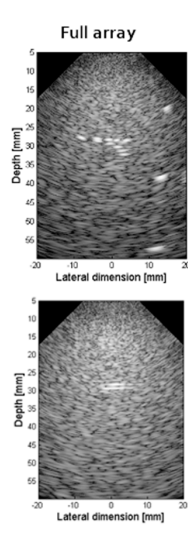

1024 elements
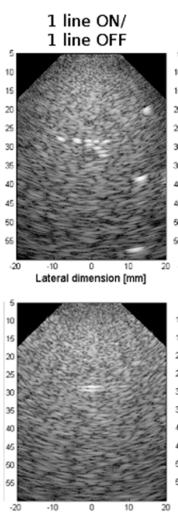

Lateral dimension [mm]

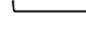

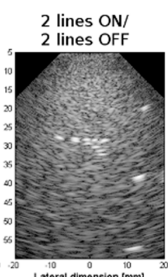
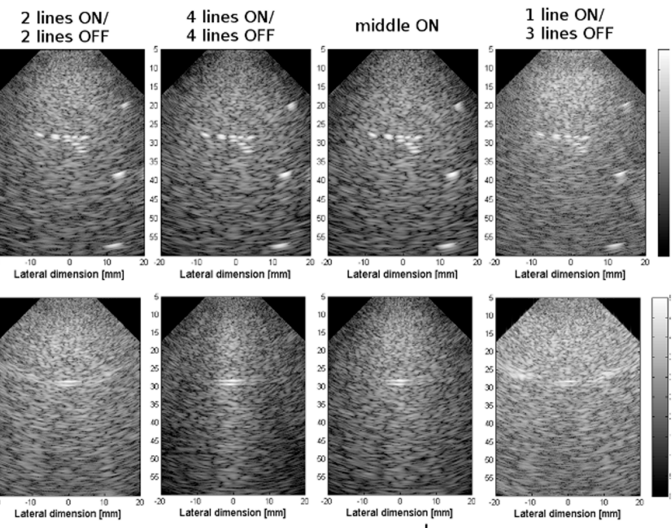

512 elements

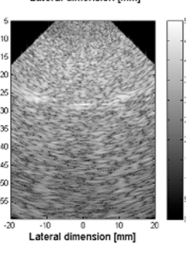

256 elements

Figure 6. Experimental data acquired with 9 DW on a "grape" wire phantom. Different configurations of the US array were used: full array, 1 line ON-1 line OFF, 2 lines ON-2 lines OFF, 4 lines ON-4 lines OFF, middle ON and 1 line ON-3 lines OFF. The shown planes $(x, z)$ (first row) and $(y, z)$ (second row) are taken in the center of the US device. A dynamic of $50 \mathrm{~dB}$ has been used in all the images.

An example of $9 \mathrm{PW}$ acquisition on a cyst phantom and wires placed at different depths is illustrated in Figure 7. In the $(x, z)$ plane, in the near field, we can identify the presented scatters (at depth $=7 \mathrm{~mm}$ and $19 \mathrm{~mm}$ ) for all the cases, but in the far-field, the contour of the cyst is affected by lower number of elements compared with the full-matrix array. The anechoic cyst at $40 \mathrm{~mm}$ depth is clearly detectable with all the configurations tested, in both $(x, z)$ and $(y, z)$ planes but its borders are less visible in $(y, z)$ planes (second row) with downsampled configurations, the degradation of the obtained images being clearly visible for cases with 512 or 256 elements. The measured values for CR and CNR obtained for each of these acquisitions in $(x, z)$ central planes are quite close for all the configurations due to the presence of the same number of active elements in the $x$ direction, while the values obtained in the $(y, z)$ plane are displayed in Table 4, the last 2 rows. Both measurements indicate the best results for the full active configuration array, followed by the configuration 4 lines ON/4 lines OFF. The worst case when considering only the configurations with 512 active elements is represented by 2 lines ON/2 lines OFF, both for CR (a value of $2.3 \mathrm{~dB}$ versus $4.2 \mathrm{~dB}$ for 4 lines $\mathrm{ON} / 4$ lines OFF) and CNR (a value of $-6.54 \mathrm{~dB}$ versus $2.61 \mathrm{~dB}$ for 4 lines $\mathrm{ON} / 4$ lines OFF). As expected, the worst results are obtained when using only 256 elements, due to the limited number of elements and important pitch between the active lines.
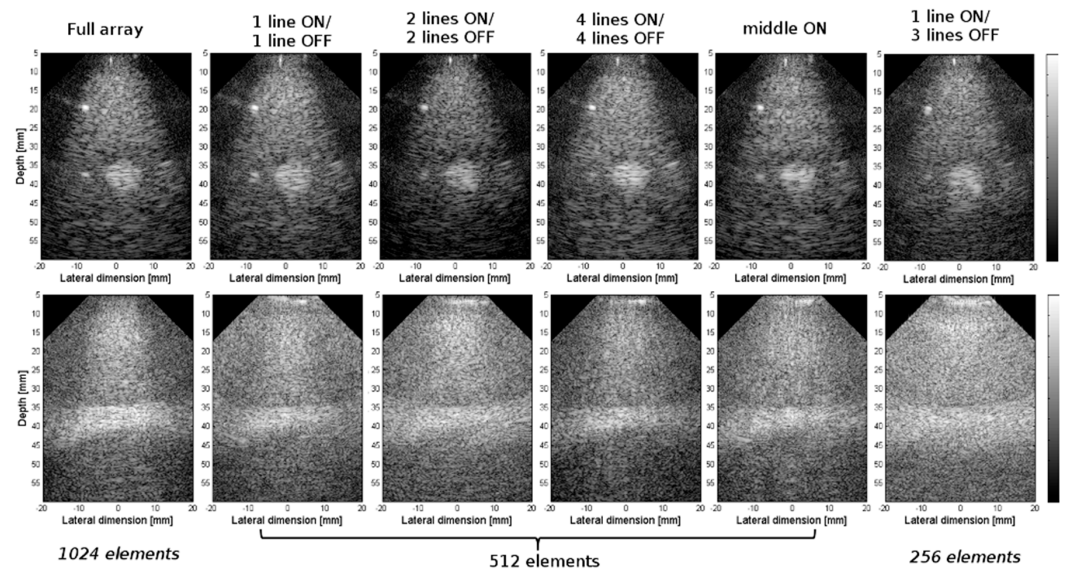

Figure 7. Experimental data acquired with $9 \mathrm{PW}$ on a standard cyst phantom. Different configurations of the US array were used: full array, 1 line ON-1 line OFF, 2 lines ON-2 lines OFF, 4 lines ON-4 lines OFF, middle ON and 1 line ON-3 lines OFF. The shown planes, $(x, z)$ (first row) and $(y, z)$ (second row) are taken in the center of the US device. A dynamic of $50 \mathrm{~dB}$ has been used in all the images. 


\subsection{Fast Volumetric Imaging for Doppler Application}

The feasibility of the 4-D platform implementation is shown here on a blood vessel mimicking phantom (described in Section 2.4) for 3-D Doppler applications. The results indicate the capability to extract the Power Doppler information when fast volumetric imaging is performed. Two different cases are presented in Figure 8 the US probe parallel (first row) and tilted by an angle of $12^{\circ}$ from the vessel phantom (second row). One PW was transmitted for each acquisition. In B-mode, the walls of the phantom are easily detected in all the planes without any distortion. The frame-rate available for volumetric acquisitions is sufficient to detect the blood mimicking fluid for power Doppler evaluation. Some artifacts are visible in the presented cases, on the distant wall of the imaged phantom.

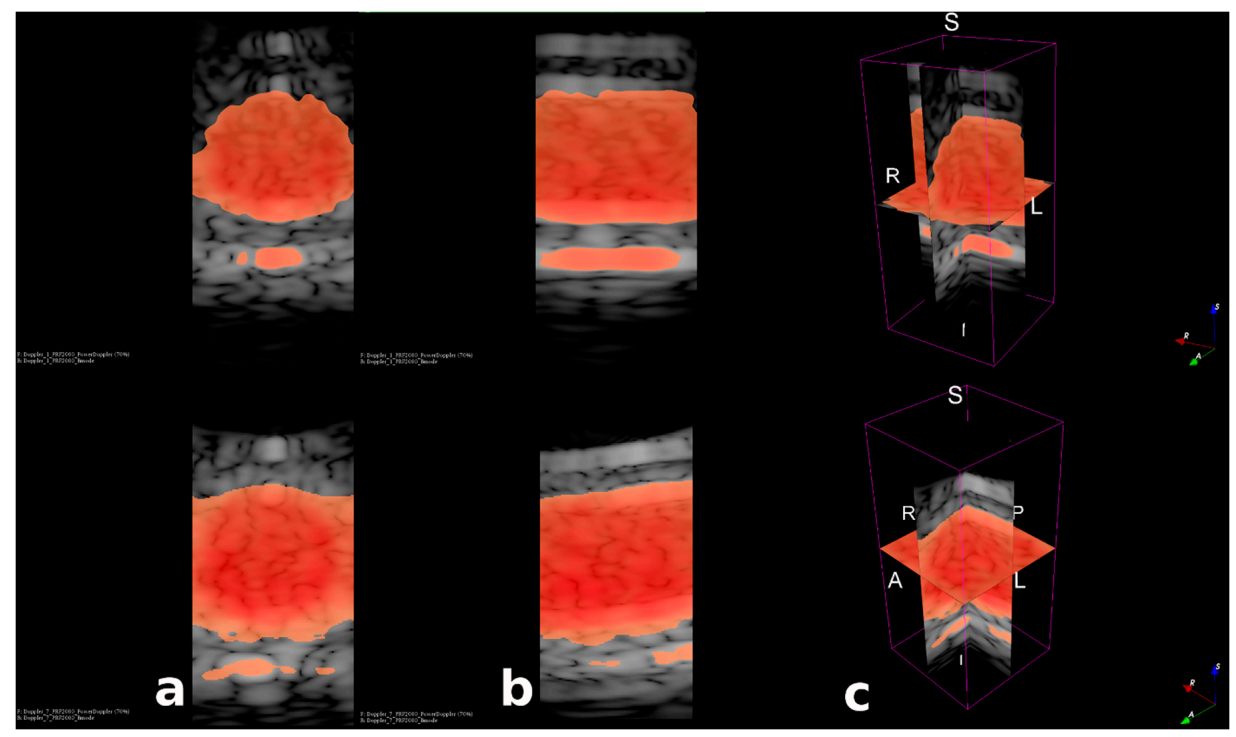

Figure 8. Examples of Power Doppler illustrations of two US acquisitions on a blood vessel mimicking phantom. The US probe is parallel $(\theta=0)$ to the phantom-tube for the first example (first row) while the probe was tilted at an angle of $12^{\circ}$ from the phantom-tube. Axial (a), lateral (b) and 3-D view rendering (c) is illustrated here. A dynamic of $50 \mathrm{~dB}$ has been used for the B-mode grayscale image.

\section{Discussion}

This study illustrates the development and feasibility of a real-time 4-D US imaging platform in full array mode by synchronizing emission and reception of up to 1024 elements independently. The initial experiments conducted in this study demonstrate the flexibility of the modular US platform, having the possibility to reconfigure it according to the number of available Vantage systems (between 1 and 4). This allows us to use full array, dense/halves/quarters of the array or to downsample (regular or sparse) the full array, as needed. Different imaging strategies were investigated using this US platform, including plane, diverging or focused waves and also high frame-rate imaging techniques. Moreover, a Doppler detection strategy was implemented as a first application by using volumetric fast imaging.

Preliminary numerical simulations indicated the image quality for some cases with full and with regular downsampled array when using the $32 \times 32$ US matrix. This allowed us to understand how the image quality will change for different configurations and to determine the best physical addressing of the US probe final design. The experimental data obtained when using the same configurations of the transducer showed the feasibility of real-time 4D US acquisitions in simultaneous mode in different systems.

As expected, the best configuration in terms of image quality was obtained experimentally when the full matrix array was active while using 4 Vantage systems. The worst configuration included only 256 elements, with only one US system was required. At the same time, this is the only configuration 
allowing processing of the data and displaying the image in real time, this issue being very important for positioning and guidance. Future work will be focused on developing strategies for increasing the image quality when using one single system, in order to be able to provide images of clinical use, especially for moving organs. The implementation in synchronous mode will follow an increase in the number of active elements, but the data must be reprocessed offline. Knowing that 3-D volume images are acquired, the data offer great utility for offline analyses even after the examination is done, reducing repeat examination and making it more cost-effective for the patient.

The integration of the four 256-channels systems into a single 1024-channels system is theoretically possible, but requires further technical developments of the constructor. Such an evolution could facilitate the transition to clinical applications, opening the possibility to collect and process the data acquired by all 1024 channels in near real-time, improving the image quality.

Overall, when inspecting the $(x, z)$ plane positioned in the center of the transducer array, no major differences were detected due to the same number of active elements (32) in the $x$ direction with all the tested configurations. When using a larger pitch (in $y$ direction) for a reduced number of elements, more important secondary lobes are visible in the $(y, z)$ plane and therefore, we have lower image contrast. Depending on the pitch value, the secondary lobes appear more or less visible, illustrating the degradation of the images. The same result is obtained when using PW and DW.

Among the compared configurations with 512 elements, in the near-field, no visual difference is observed when using the same number of elements, while in the far-field and on the lateral parts of the images, the contours are seriously affected when using only the central part of the US probe (see Figures 5 and 7). In addition, when evaluating the FWHM, the configuration with 2 lines ON/2 lines OFF and 1 line ON/1 line OFF seems to be the closer to the 1024 elements, but the measured values for all these configurations remain quite close.

Moreover, a lower transmit energy compromises the image quality by diminishing the $\mathrm{CR}$ and $\mathrm{CNR}$, from $7.71 \mathrm{~dB}(\mathrm{CR})$ and $2.99 \mathrm{~dB}(\mathrm{CNR})$, when using full active array, to $2.02 \mathrm{~dB}(\mathrm{CR})$ and $-7.31 \mathrm{~dB}$ (CNR), respectively, for the configuration with 256 active elements. The results obtained experimentally are in good agreement with preliminary numerical simulations, and the image degradation is explained by the different values of the pitch in the $y$ direction.

By using the presented 4-D US platform, the power Doppler technique was exploited to detect moving particles. Here, only the full configuration array was used to prove the feasibility of the method, the experiments with lower number of elements being beyond the scope of this article. This method is particularly useful when examining superficial structures. The capability of obtaining entire volumes at a high frame-rate (used PRF acquisitions on blood vessel mimicking phantom was $2000 \mathrm{~Hz}$ ) opens a wide field of vascular and cardiac applications. The feasibility of the ultrafast imaging is very important in the context of cardiac imaging. Here, the difficulty is the heart beating. Therefore, the high frame-rate allows performing the acquisition in less time in order to not be disurbed by heart movement [23].

The combination of hardware and software-based technologies provides full flexibility of the developed platform, its reconfgurability being one of its major advantages. This makes it suitable for different US applications requiring 1024 channels or less in regular or optimized sparse arrays. As the US scanners are completely open systems, our platform can be programmed to support different strategies which typically cannot be implemented in conventional data flow architectures. Different methods can be investigated: novel transmission and/or reception methods and sequences, apodization windowing [35], non-conventional beamforming techniques, excitation amplitude and aperture control, etc., but also custom-data processing. Such a platform will indeed be crucial to objectivize the real adjunct and efficiency of advanced reconstruction strategy, aiming at providing similar image quality as from highly undersampled acquisition since they can be compared against ground truth available for a full configuration. Indeed, over-performing methods are often published without a true reference implementation of the acquisition, and data are generally virtually undersampled, lacking realism. 
The connectors of the US probe were chosen in order to allow adapting the impedance while for all 4 systems we have the possibility to have the HIFU (High Intensity Focused Ultrasound) option. Different studies have already illustrated the possibility to use HIFU for pre-clinical and clinical cardiac applications such as: treatment of arrhythmias [36,37], atrial septostomy [38] or atrio-ventricular nodal-ablation in dogs [39]. Therefore, the developed 4-D US platform can be considered as dual-mode, imaging and therapy. This aspect can be exploited, even using low intensity therapies (the US probe presented in this manuscript is not developed for HIFU mode), for theragnostic applications in the heart, for example. Here, the major interest is represented by the use of the same system for imaging and therapy.

One of the technical limitations of this platform when using the described US probe involves the physical addressing of the elements. When using 512 active elements in configurations "middle $\mathrm{ON}^{\prime}$ " and "4 lines OFF/4 lines ON", we still need 4 synchronized US systems even if not all the channels are used. This issue could be improved by using US probes with a different addressing connectivity.

The next technical step includes technical improvements for near real-time visualization of data received by several systems: high computational power and storage capability will be required. Therefore, the large acquired data flow will be rapidly transferred, stored and processed, the complementary information provided by the 4 systems being visualized immediately.

By using this powerful US platform, the advantages offered by a 2-D probe with a high number of channels can be easily investigated. Moreover, the flexibility of the system allows for implementing a large class of US applications, in therapy or monitoring. The technical feasibility of real-time 4-D low energy US for imaging and potentially for therapeutic purposes was confirmed with this 1024-channels high-density US system, offering full research access for developing advanced US strategies for different applications.

\section{Conclusions}

A new powerful high-channels US platform for implementing 4-D (real-time 3-D) advanced US strategies, offering full research access, was developed and presented here. The conducted experiments demonstrate its technical feasibility and flexibility, allowing us to use a full or downsampled array, as needed, illustrating the degree of degradation of image quality for each combination. Different imaging strategies were investigated using this US platform, and a Doppler detection strategy was implemented as a first application by using volumetric fast imaging, opening a large field of cardiac and vascular applications, in therapy or monitoring.

Supplementary Materials: The following are available online at www.mdpi.com/2076-3417/8/2/200/s1. The following Supplementary Materials are available online: Animation of the 3-D rendering of Power Doppler obtained when the US probe is parallel to the vessel phantom, corresponding to the example illustrated in Figure 8, first row. Animation of the 3-D rendering of Power Doppler obtained when the US probe is tilted from the vessel phantom corresponding to the example illustrated in Figure 8, second row.

Acknowledgments: This work was completed as part of the framework of the "Programme Avenir Lyon Saint-Etienne" of the Université de Lyon (ANR-11-IDEX-0007), within the Program "Investissements d'Avenir" operated by the French National Research Agency and co-founded by the People Programme (Marie Curie Actions) of the European Union's Seventh Framework Programme (FP7/2007-2013) under REA grant agreement $\mathrm{n}^{\circ}$ PCOFUND-GA-2013-609102, through the PRESTIGE programme coordinated by Campus France. It was also performed within the framework of the Labex PRIMES (ANR-10-LABX-0063) of Universite de Lyon, within the program "Investissements d'Avenir" (ANR-11-IDEX-0007) operated by the French Nation-al Research Agency (ANR). 2 Verasonics systems were cofounded by the FEDER program, Saint-Etienne Metropole (SME) and Conseil General de la Loire (CG42) within the framework of the SonoCardioProtection Project leaded by Pr Pierre Croisille. The authors would also like to thank LabTAU for their contribution to the development of the $32 \times 32$ probe prototype compatible with driving 1 to 4 systems as well as for the provision of the probe and two Vantage 256 systems.

Author Contributions: L.P., F.V. W.A.N. designed and carried out the simulations and experimental studies; L.P. and F.V. analyzed the data; R.S. and A.B. provided technical support; J.Y.C., H.L. W.A.N. and M.V. provided critical feedback for the different steps of this project ; L.P. wrote the original draft. All co-authors contributed with reading and improving the final manuscript.

Conflicts of Interest: The authors declare no conflict of interest. 


\section{References}

1. Tortoli, P.; Bassi, L.; Boni, E.; Dallai, A.; Guidi, F.; Ricci, S. ULA-OP: An advanced open platform for ultrasound research. IEEE Trans. Ultrason. Ferroelectr. Freq. Control 2009, 56, 2207-2216. [CrossRef]

2. Asef, A.A.; Maia, J.M.; Costa, E.T. A flexible multichannel FPGA and PC-Based ultrasound system for medical imaging research: Initial phantom experiments. Res. Biomed. Eng. 2015, 31, 277-281.

3. Brunke, S.S.; Insana, M.F.; Dahl, J.J.; Hansen, C.; Ashfaq, M.; Ermert, H. An ultrasound research interface for a clinical system. IEEE Trans. Ultrason. Ferroelectr. Freq. Control 2007, 54, 198-210. [CrossRef]

4. Roux, E.; Varray, F.; Petrusca, L.; Cachard, C.; Tortoli, P.; Liebgott, H. 3D diverging waves with 2D sparse arrays: A feasibility study. In Proceedings of the IEEE International Ultrasonics Symposium, Washington, DC, USA, 6-9 September 2017.

5. Roux, E.; Ramalli, A.; Tortoli, P.; Cachard, C.; Robini, M.; Liebgott, H. 2D ultrasound sparse arrays multi-depth radiation optimization using simulated annealing and spiral-array inspired energy functions. IEEE Trans. Ultrason. Ferroelectr. Freq. Control 2016, 63, 2138-2149.

6. Badescu, E.; Bujoreanu, D.; Petrusca, L.; Friboulet, D.; Liebgott, H. Multi-line transmission for 3D ultrasound imaging: An experimental study. In Proceedings of the IEEE International Ultrasonics Symposium, Washington, DC, USA, 6-9 September 2017.

7. Zhang, M.; Varray, F.; Besson, A.; Carrillo, R.E.; Viallon, M.; Garcia, D.; Thiran, J.P.; Friboulet, D.; Liebgott, H.; Bernard, O. Extension of fourier-based techniques for ultrafast imaging in ultrasound with diverging waves. IEEE Trans. Ultrason. Ferroelectr. Freq. Control 2016, 63, 2125-2137. [CrossRef]

8. Salles, S.; Liebgott, H.; Garcia, D.; Vray, D. Full 3D transverse oscillations: A method for tissue motion estimation. IEEE Trans. Ultrason. Ferroelectr. Freq. Control 2015, 62, 1473-1485. [CrossRef]

9. Tanter, M.; Fink, M. Ultrafast imaging in biomedical ultrasound. IEEE Trans. Ultrason. Ferroelectr. Freq. Control 2014, 61, 102-119. [CrossRef]

10. Synnevag, J.-F.; Austeng, A.; Holm, G. Adaptive beamforming applied to medical ultrasound imaging. IEEE Trans. Ultrason. Ferroelectr. Freq. Control 2007, 54, 160-1613. [CrossRef]

11. Provost, J.; Papadacci, C.; Arango, J.E.; Imbault, M.; Fink, M.; Gennisson, J.L.; Tanter, M.; Pernot, M. 3D ultrafast ultrasound imaging in vivo. Phys. Med. Biol. 2014, 59, L1-L13. [CrossRef]

12. Jensen, J.A.; Holten-Lund, H.; Nilsson, R.T.; Hansen, M.; Larsen, U.D.; Domsten, R.P.; Tomov, B.G.; Stuart, M.B.; Nikolov, S.I.; Pihl, M.J.; et al. SARUS: A synthetic aperture real-time ultrasound system. IEEE Trans. Ultrason. Ferroelectr. Freq. Control 2013, 60, 1838-1852. [CrossRef]

13. Provost, J.; Papadacci, C.; Demene, C.; Gennisson, J.L.; Tanter, M.; Pernot, M. 3-D ultrafast doppler imaging applied to the noninvasive mapping of blood vessels in vivo. IEEE Trans. Ultrason. Ferroelectr. Freq. Control 2015, 62, 1467-1472. [CrossRef] [PubMed]

14. Papadacci, C.; Finel, V.; Provost, J.; Villemain, O.; Bruneval, P.; Gennisson, J.L.; Tanter, M.; Fink, M.; Pernot, M. Imaging the dynamics of cardiac fiber orientation in vivo using 3D Ultrasound Backscatter Tensor Imaging. Sci. Rep. 2017, 7, 830. [CrossRef] [PubMed]

15. Savord, B.; Solomon, R. Fully sampled matrix transducer for real time 3D ultrasonic imaging. In Proceedings of the IEEE Symposium on Ultrasonics, Honolulu, HI, USA, 5-8 October 2003; Volume 1, pp. 945-953.

16. Bhuyan, A.; Choe, J.W.; Lee, B.C.; Wygant, I.O.; Nikoozadeh, A.; Oralkan, Ö.; Khuri-Yakub, B.T. Integrated circuits for volumetric ultrasound imaging with 2-D CMUT arrays. IEEE Trans. Biomed. Circuits Syst. 2013, 7, 796-804. [CrossRef] [PubMed]

17. Kortbek, J.; Jensen, J.A.; Gammelmark, K.L. Sequential beamforming for synthetic aperture imaging. Ultrasonics 2013, 53, 1-16. [CrossRef] [PubMed]

18. Christiansen, T.L.; Rasmussen, M.F.; Bagge, J.P.; Nordahl Moesner, F.; Jensen, J.A.; Thomsen, E.V. 3-D imaging using row-column-addressed arrays with integrated apodization-Part II: Transducer fabrication and experimental results. IEEE Trans. Ultrason. Ferroelectr. Freq. Control 2015, 62, 959-971. [CrossRef] [PubMed]

19. Didier, D.; Brusseau, E.; Detti, V.; Varray, F.; Basarab, A. Ultrasound medical imaging. In Medical Imaging Based on Magnetic Fields and Ultrasounds; John Wiley \& Sons: Hoboken, NJ, USA, 2014; pp. 1-72.

20. Papadacci, C.; Pernot, M.; Couade, M.; Fink, M.; Tanter, M. High-contrast ultrafast imaging of the heart. IEEE Trans. Ultrason. Ferroelectr. Freq. Control 2014, 61, 288-301. [CrossRef] [PubMed]

21. Hasegawa, H.; Kanai, H. High-frame-rate echocardiography using diverging transmit beams and parallel receive beamforming. J. Med. Ultrason. 2011, 38, 129-140. [CrossRef] 
22. Tong, L.; Ramalli, A.; Tortoli, P.; Fradella, G.; Caciolli, S.; Luo, J.; D’hooge, J. Wide-angle tissue doppler imaging at high frame rate using multi-line transmit beamforming: An experimental validation in vivo. IEEE Trans. Med. Imaging 2016, 35, 521-528. [CrossRef]

23. Poree, J.; Posada, D.; Hodzic, A.; Tournoux, F.; Cloutier, G.; Garcia, D. High-frame-rate echocardiography using coherent compounding with doppler-based motion-compensation. IEEE Trans. Med. Imaging 2016, 35, 1647-1657. [CrossRef]

24. Joos, P.; Liebgott, H.; Varray, F.; Petrusca, L.; Garcia, D.; Vray, D.; Nicolas, B. High-frame-rate 3-D echocardiography based on motion compensation: An in vitro evaluation. In Proceedings of the IEEE International Ultrasonics Symposium, Washington, DC, USA, 6-9 September 2017.

25. Cikes, M.; Tong, L.; Sutherland, G.R.; D’hooge, J. Ultrafast cardiac ultrasound imaging. JACC Cardiovasc. Imaging 2014, 7, 812-823. [CrossRef]

26. Vappou, J.; Luo, J.; Konofagou, E.E. Pulse wave imaging for noninvasive and quantitative measurement of arterial stiffness in vivo. Am. J. Hypertens. 2010, 23, 393-398. [CrossRef] [PubMed]

27. Bercoff, J.; Montaldo, G.; Loupas, T.; Savery, D.; Mézière, F.; Fink, M.; Tanter, M. Ultrafast compound Doppler imaging: Providing full blood flow characterization. IEEE Trans. Ultrason. Ferroelectr. Freq. Control 2011, 58, $134-147$. [CrossRef] [PubMed]

28. Jensen, J.A. Field: A program for simulating ultrasound systems. In Proceedings of the 10th Nordic-Baltic Conference on Biomedical Imaging Published in Medical \& Biological Engineering \& Computing, Tampere, Finland, 9-13 June 1996; Volume 34, pp. 351-353.

29. Jensen, J.A.; Svendsen, N.B. Calculation of pressure fields from arbitrarily shaped, apodized, and excited ultrasound transducers. IEEE Trans. Ultrason. Ferroelectr. Freq. Control 1992, 39, 262-267. [CrossRef] [PubMed]

30. Perrot, V.; Petrusca, L.; Bernard, A.; Vray, D.; Liebgott, H. Simultaneous pulse wave and flow estimation at high-framerate using plane wave and transverse oscillation on carotid phantom. In Proceedings of the IEEE International Ultrasonics Symposium, Washington, DC, USA, 6-9 September 2017.

31. Fromageau, J.; Gennisson, J.L.; Schmitt, C.; Maurice, R.; Mongrain, R.; Cloutier, G. Estimation of polyvinyl alcohol cryogel mechanical properties with four ultrasound elastography methods and comparison with gold standard testings. IEEE Trans. Ultrason. Ferroelectr. Freq. Control 2007, 54, 498-509. [CrossRef] [PubMed]

32. Ramnarine, K.V.; Nassiri, D.K.; Hoskins, P.R.; Lubbers, J. Validation of a new blood-mimicking fluid for use in doppler flow test objects. Ultrasound Med. Biol. 1998, 24, 451-459. [CrossRef]

33. Montaldo, G.; Tanter, M.; Bercoff, J.; Benech, N.; Fink, M. Coherent plane-wave compounding for very high frame rate ultrasonography and transient elastography. IEEE Trans. Ultrason. Ferroelectr. Freq. Control 2009, 56, 489-506. [CrossRef] [PubMed]

34. Fedorov, A.; Beichel, A.; Kalpathy-Cramer, J.; Finet, J.; Fillion-Robin, J.C.; Pujol, S.; Bauer, C.; Jennings, D.; Fennessy, F.; Sonka, M.; et al. 3D slicer as an image computing platform for the Quantitative Imaging Network. Magn. Reson. Imaging 2012, 30, 1323-1341. [CrossRef] [PubMed]

35. Tomov, B.G.; Jensen, J.A. Compact implementation of dynamic receive apodization in ultrasound scanners. In Medical Imaging 2004: Ultrasonic Imaging and Signal Processing; SPIE: San Diego, CA, USA, 2004.

36. Ninet, J.; Roques, X.; Seitelberger, R.; Deville, C.; Pomar, J.L.; Robin, J.; Jegaden, O.; Wellens, F.; Wolner, E.; Vedrinne, C.; et al. Surgical ablation of atrial fibrillation with off-pump, epicardial, high-intensity focused ultrasound: Results of a multicenter trial. J. Thorac. Cardiovasc. Surg. 2005, 130, 803-809. [CrossRef] [PubMed]

37. Bessiere, F.; N'djin, W.A.; Colas, E.C.; Chavrier, F.; Greillier, P.; Chapelon, J.Y.; Chevalier, P.; Lafon, C. Ultrasound-guided transesophageal high-intensity focused ultrasound cardiac ablation in a beating heart: A pilot feasibility study in pigs. Ultrasound Med. Biol. 2016, 42, 1848-1861. [CrossRef] [PubMed]

38. Takei, Y.; Muratore, R.; Kalisz, A.; Okajima, K.; Fujimoto, K.; Hasegawa, T.; Arai, K.; Rekhtman, Y.; Berry, G.; Homma, S.; et al. In vitro atrial septal ablation using high-intensity focused ultrasound. J. Am. Soc. Echocardiogr. 2012, 25, 467-472. [CrossRef] [PubMed]

39. Strickberger, S.A.; Tokano, T.; Kluiwstra, J.U.; Morady, F.; Cain, C. Extracardiac ablation of the canine atrioventricular junction by use of high-intensity focused ultrasound. Circulation 1999, 100, 203-208. [CrossRef] [PubMed]

(C) 2018 by the authors. Licensee MDPI, Basel, Switzerland. This article is an open access article distributed under the terms and conditions of the Creative Commons Attribution (CC BY) license (http://creativecommons.org/licenses/by/4.0/). 